\title{
The future of combustion engines from the perspective of rail applications
}

Andreas Wegmann, M. Gayer

J. M. Voith SE \& Co. KG | VTA

B. Gay, M. Pister

Liebherr Machines Bulle SA

This manuscript is not available according to publishing restriction.

Thank you for your understanding. 